\title{
Physical training improves the spleen redox situation in response to the stress of a bout of physical exercise in genetically obese rats
}

\author{
N. M. De Castro ${ }^{1}$, I. Baeza ${ }^{1}$, C. Vida ${ }^{1}$, O. Hernández ${ }^{1}$, R. Manso ${ }^{2}$ and M. De La Fuente ${ }^{1}$ \\ ${ }^{1}$ Department of Physiology, Faculty of Biology, Complutense University of Madrid, 28040 Madrid, Spain and ${ }^{2}$ Department \\ of Molecular Biology, Faculty of Sciences, Autonomous University of Madrid, 28049 Madrid, Spain
}

Obesity is characterised by a low-grade inflammation of white adipose tissue ${ }^{(1)}$, and inflammatory conditions are associated with an increase of oxidative stress ${ }^{(2)}$. There is evidence that regular exercise, of moderate intensity and duration, up-regulates the immune system and induces a decreased of oxidative stress acting as a hormetic response. Therefore, the aim of the present work is to study the effects of training exercise on several oxidative stress parameters in spleen and liver, in a murine model of genetic obesity. Male obese Zucker ('fa/fa') rats were divided into four groups: sedentary, submitted to moderate training ( 1 daily treadmill-running session for 16 consecutive weeks; speed and duration increased gradually), and sedentary and trained groups which were submitted to a bout of exercise (treadmillrunning for $30 \mathrm{~min}$ immediately before sacrifice). All animals were fed standard diet (PANLAB A04). At 6 months of age all groups were sacrificed and homogenates from spleen and liver were obtained. The oxidative stress parameters analysed were: xanthine oxidase activity (XO), as an oxidant parameter, as well as total antioxidant capacity (TAC), total gluthatione (GSH) and gluthatione reductase (GR) activity as antioxidant defence parameters. The results showed that XO activity increased, in both spleen and liver, after a bout of physical exercise in sedentary but not in trained animals. The total antioxidant capacity decreased in the sedentary rats submitted to a bout of exercise in spleen and liver, but not in trained animals. The GSH levels were increased in trained group submitted to a bout of exercise with respect to the trained group in spleen. Thus, the training programme seems to prepare the animals for the stressful bout of exercise, increasing this important antioxidant parameter. Furthermore, the results showed that this increase was not caused by an increase in the GR activity, since this antioxidant activity did not show any changes in spleen and liver. Nevertheless, the GSH increase could be consequence of an increase of its synthesis in liver by training exercise, since the levels of this antioxidant increase in this group with respect to sedentary animals. In conclusion, a bout of exercise is a physical stress that increases oxidants and decreases the antioxidant capacity, causing an oxidative stress situation in sedentary rats, but not in those submitted to the physical training programme. Training exercise results in a better redox condition and prepares Zucker rats, increasing the level of antioxidant defences that produce the organism, for a better response to that physical stress.

Xo spleen

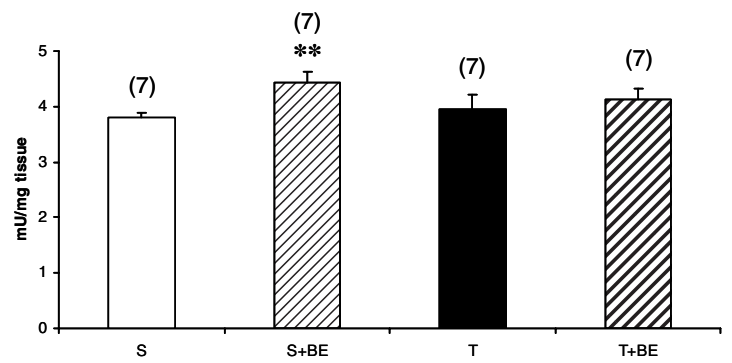

Total antioxidant capacity spleen

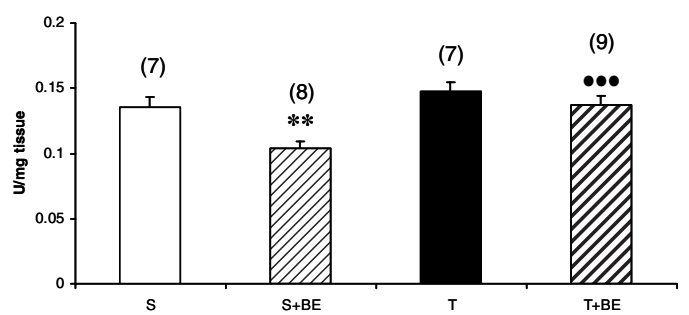

Data are expressed as means $\pm \mathrm{SE}$ of the number of animals used (in brackets). $\mathrm{S}$, sedentary group; $\mathrm{T}$, trained group; $\mathrm{S}+\mathrm{BE}$, sedentary + bout exercise; $\mathrm{T}+\mathrm{BE}$, trained + bout exercise.

$* * P<0.01$ with respect to sedentary group. $\bullet \bullet P<0.001$ with respect to sedentary + bout exercise group.

This work was financially supported by MEC (DEP2006-56187-CO-02/PREV), UCM Research Group (910379ENEROINN) and RETICEF (RD06/0013/ 0003) (ISCIII) of Spain.

1. Bastard JP, Maachi M, Lagathu C et al. (2006) Eur Cytokine Netw 17, 4-12.

2. De la Fuente M (2008) Neuroimmunomodulation 15, 213-223. 